\title{
Enhanced Power Control MAC Protocol for Single Cell, Dense Wireless Ad Hoc Networks
}

\author{
Sofy Harold \\ M.Tech, Final Year \\ Department of Electronics and Communication \\ Engineering \\ Sri Manakula Vinayagar Engineering College, \\ Madagadipet, Puducherry-605017
}

\author{
Vijayalakshmi.A \\ Associate Professor \\ Department of Electronics and Communication \\ Engineering \\ Sri Manakula Vinayagar Engineering College, \\ Madagadipet, Puducherry-605017
}

\begin{abstract}
A new reliable protocol called Enhanced Power Control MAC Protocol for Wireless Ad- Hoc Networks (EPCMAC) is proposed in this project work. The key concept of this EPCMAC protocol is to improve the throughput, transport capacity and to save energy by sending all the packets with optimal transmit power. This communication approach promises improved throughput and delay performance by effective use of spatial diversity in wireless ad hoc networks. Also, the power of the data packets is periodically raised to a suitable level but not to the maximum so that it will avoid interference and unnecessary contention between nodes. Throughput enhancement of a wireless ad hoc networking at the MAC layer is achieved through spatial reuse of the channel by allowing concurrent transmissions as much as possible among neighboring nodes. Simulation results show that a considerable gain in throughput as well as high reduction in energy consumption can be obtained by EPCMAC protocol compared to the existing protocols.
\end{abstract}

\section{Keywords}

IEEE 802.11, EPCMAC, MAC protocol, PCM, OHOP,

\section{Ad hoc networks.}

\section{INTRODUCTION}

Mobile ad hoc networks (MANET) continue to be a topic of increasing interest to the wireless communication community. It can be considered an emerging fourth-generation (4G) wireless system that supports anytime, anywhere and from any device communication. Ad hoc networks are infrastructure less such that they can operate without the support of centralized entities and therefore can be quickly deployed in a given area and provide robust operation. Currently, the applications of ad hoc networks that have drawn considerable attention relate to military, emergency services, sensor networks and home networking, however, more mainstream applications relating to business, education, entertainment, and commercial services are quite viable. The following figure is an illustration of ad hoc networking.

The transmission of nodes that exchanged RTS-CTS successfully, may collide with DATA/ACK transmission of other nodes that had not overheard the RTCCTS. Several drawbacks of IEEE 802.11 have been identified in the past several years. IEEE 802.11 uses maximum transmission power Pmax regardless of the distance between the transmitter and receiver. This gives inefficient use of energy, since a successful communication between a transmitter and receiver pairs with short distance is possible with much lesser power than Pmax . Most power control schemes for wireless Ad Hoc networks have been proposed to reduce the energy consumption for increasing the life- time of the network.

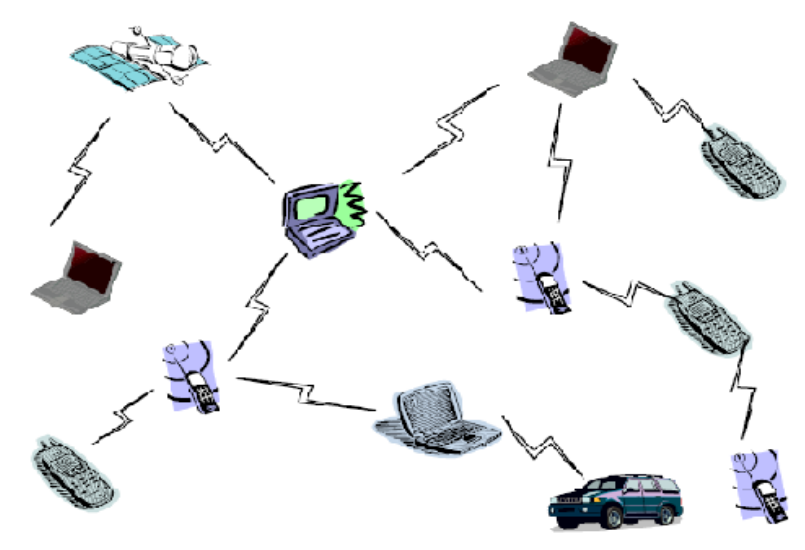

Fig 1: an example of ad hoc network

However these schemes may increase energy consumption due to the increase in interference range. Other power control schemes that have been proposed to improve energy efficiency do this at the cost of throughput.

In this paper, we proposed a new power control protocol which simultaneously improves the throughput and yield energy saving. The rest of this paper is organized as follows. Section 2 reviews the related work. Our proposed EPCMAC protocol is explained in section 3. Section 4 presents simulation and experimental results. Finally, section 5 concludes the work presented in this paper.

\section{EXISTING WORK}

A power control mechanism that can be incorporated into the IEEE 802.11 RTS-CTS handshake. The scheme in allows a node, A, to specify its current transmit power level in the transmitted RTS, and allows receiver node B to include a desired transmit power level in the CTS sent back to A. On receiving the CTS, node A then transmits DATA using the power level specified in the CTS. This scheme allows B to help A choose the appropriate power level, so as to maintain a desired signal-to-noise ratio. A similar protocol is utilized wherein the RTS and CTS packets are sent at the highest power level, and the DATA and ACK may be sent at a lower power level. We refer to this scheme as the BASIC power 
control MAC protocol. The BASIC scheme has a shortcoming that can degrade the throughput. Furthermore, the BASIC scheme may potentially increase the energy consumption, instead of decreasing it.

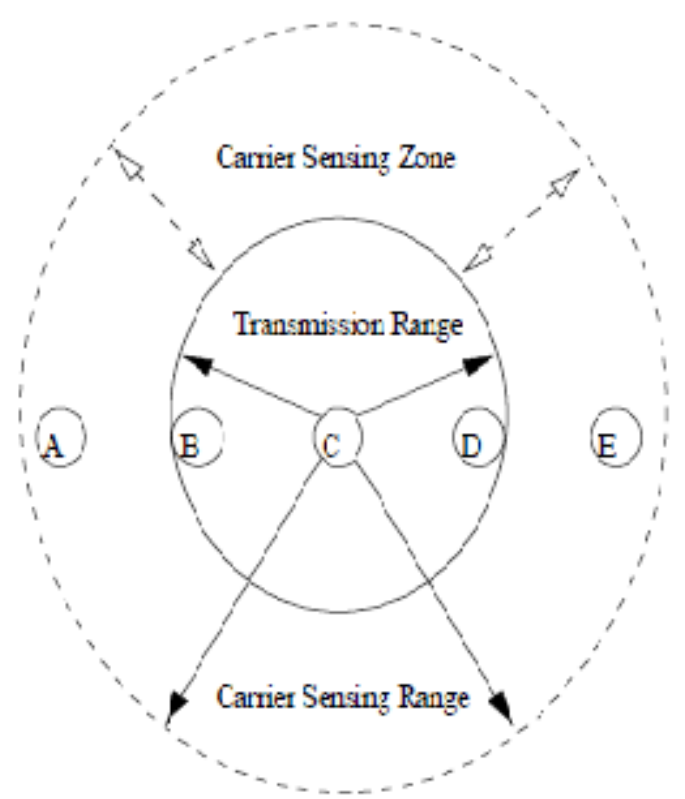

Fig 2: nodes in transmission range

This figure 2 describes that PARO a power-aware routing optimization, determines routes which consume low energy. PARO chooses a cost function based on the transmit power level at each hop on a route, to determine a low energyconsuming route between a pair of nodes. PARO also uses a power control MAC protocol similar to BASIC. A power control protocol presented in the BASIC scheme. It maintains a table for the minimum transmits power necessary to communicate with neighbor nodes. This scheme allows each node to increase or decrease its power level dynamically. However, different power levels among nodes result in asymmetric links, causing collisions.

\section{ENHANCED POWER CONTROL MAC PROTOCOL FOR WIRELESS AD HOC NETWORK}

\subsection{Background of Proposed Protocol}

In PCM protocol, collision avoidance is generalized to power control. Conventional collision avoidance methods had an "on/off model", wherein a node can either transmit (if it is not deferring and does not sense a busy carrier) or not. However, a node can transmit to its intended receiver so long as it satisfies four constraints. Thus, the on/off model is generalized to a bounded-power mode. In order to achieve the bounded- power model, the power control component in PCMA has two main mechanisms:

Proposed EPCMAC protocol is similar to the PCM scheme except that the source node transmits DATA with the optimum power level. This power level is periodically increased, for just enough time not to Pmax as in PCM, but to a suitable level (Pai) sufficient to avoid the collisions. The EPCMAC protocol can be considered as an improved version of PCM protocol. PCM transmit the data with maximum periodic pulse power. This means reserving maximum transmission area for the giving ongoing transmission even the distance between the transmitter and receiver is small. The objective behind using maximum periodic pulse power is to increase the sensing range for informing the neighbour nodes about ongoing transmission in order to reduce the interference and increase energy conservation.

But, increasing the carrier sensing range to maximum range affects the total throughput of the network, since some nodes in the maximum carrier sensing range can also transmit data successfully to its corresponding receiver without affecting the first ongoing transmission.

For example, in a chain topology of 31 nodes with 30 flows and the distance between adjacent node pairs is $40 \mathrm{~m}$, the carrier sensing range of $134 \mathrm{~m}$ is enough to avoid interference compared to $550 \mathrm{~m}$ as in the case of PCM. Let Rtop be the transmission range of the RTS using the optimum power. Since RTS and CTS use the same optimum power, the transmission range of CTS will be Rtop also. Suppose that the periodic pulse power is also the same (i.e optimum), the carrier sensing range is at least twice times the transmission range.

If the receiver is just at the edge of the transmission range of the transmitter, carrier sensing range $(R c s)$ will cover both transmission ranges of RTS and CTS as shown in Fig.1. Actually, this case is considered as the worst case. Usually, the periodic pulse power will be greater than the optimum used power. It depends on the distance between the transmitter and the receiver for any optimum power lesser than the maximum power. This means, the ongoing transmission will be completely covered by the carrier sensing range. Any node in the carrier sensing range, but not in the RTS or CTS range will notice the transmission and therefore will defer its transmission request. According to IEEE 802.11DCF, this node will maintain a NAV.

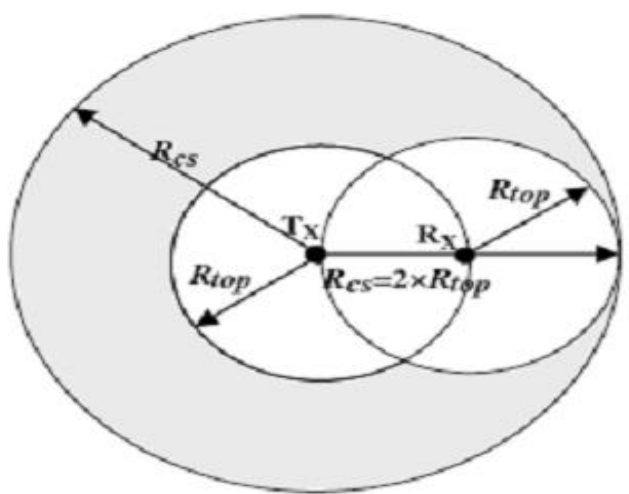

Fig 3: the carrier sensing range

\subsection{Calculation of Signal to Interference Ratio (SIR) Value}

Radio engineers typically use a model that attenuates the power of signal as $1 / d 2$ at short distances (free space propagation model) and as $1 / d 4$ at longer distances (two-ray ground reflection model), where $d$ is the distance between antennas. The crossover point is called the reference distance. Therefore, the signal propagation model used in our work is a combination of the free space propagation model (for distances less than the reference distance) and the two-ray ground reflection model (for distances greater than the reference distance). At near distance, the power received $(P r)$ is given by

$$
\mathcal{P}_{r}=\mathcal{P}_{t} G_{t} G_{r}\left(\frac{\lambda}{4 \pi d}\right)^{2}
$$


Where $\mathrm{Pt}$ is the transmitted power, Gt and Gr are the antenna gain of the transmitter and the receiver, respectively, $\lambda$ is the signal wavelength, and $d$ is the distance between the transmitter and the receiver.On the other hand, the power received $(\mathrm{Pr})$ at far distance is given by

$$
\mathcal{P}_{r}=\mathcal{P}_{t} G_{t} G_{r}\left(\frac{h_{t} h_{r}}{d^{2}}\right)^{2}
$$

Where $h t$ and $h r$ are the antenna height of the transmitter and the receiver, respectively. From Eq.(1) and Eq.(2), dcrossover can be derived, which isconsidered as the crossing edge from the near to far distances. This distance is given by

$$
d_{\text {crossover }}=4 \pi \frac{h_{t} h_{r}}{\lambda}
$$

The path loss $(P l,($ dcrossover $))$ at the distance dcrossover is considered as the reference value in our model. This value is constant (C) that depends on the antenna gains, the wavelength, the antenna heights and the crossover distance dcrossover.

$$
\begin{gathered}
\mathcal{P}_{t}\left(d_{\text {crossover }}\right)=\frac{\mathcal{P}_{r}\left(d_{\text {crossover }}\right)}{\mathcal{P}_{t}\left(d_{\text {crossover }}\right)}=G_{t} G_{r}\left(\frac{\lambda}{4 \pi d_{\text {crossover }}}\right)^{2} \\
=G_{t} G_{r}\left(\frac{h_{t} h_{r}}{d_{\text {crossover }}^{2}}\right)^{2}=C
\end{gathered}
$$

From Eq.(1), Eq.(2) and Eq.(3), the power received (Pr) at any distance can be rewritten in its general form as given below

$$
\mathcal{P}_{r}=\mathcal{P}_{t} C\left(\frac{d_{\text {crossover }}}{d}\right)^{\alpha}
$$

Where $\alpha$ is the path loss exponent, $\alpha=2$ in case of free space propagation model and its value is 4 in case of two ray propagation model. Let $\mathrm{Pi}$ represents the transmission power of an interfering node at distance $d i$ from a receiver. Since this interfering node will be at a distance at least equal to the carrier sensing range, it will be considered as a far distance. The receiving power Pri of the signal from the interference node will be calculated as follows:

$$
\mathcal{P}_{r i}=\mathcal{P}_{i} G_{t} G_{r}\left(\frac{h_{t} h_{r}}{d_{i}^{2}}\right)^{2}
$$

Therefore the $S I R$ value is given by

$$
\mathcal{S I R}=\frac{\mathcal{P}_{t}}{\mathcal{P}_{r i}}=\frac{\mathcal{P}_{t} C}{\mathcal{P}_{i} G_{t} G_{r}} * \frac{\left(d_{\text {crossover }} / d\right)^{\alpha}}{\left(h_{t} h_{r} / d_{i}^{2}\right)^{2}}
$$

The proposed EPCMAC protocol works in the following steps:

- Transmitter sends an RTS with the optimum transmit power level including the level of that power as shown in algorithm.

- Receiver decodes the RTS, find the power level value, observe the SIR value, attach the SIR value to the CTS packet and transmit CTS using the same optimum power.
- Transmitter extract the SIR value and sends the DATA with the optimum power, and periodically increases the power level of the DATA packets to Pai to avoid interference. The $P a i$ value is selected

based on the ratio of the channel capacity and the carrier sensing range.

- The receiver sends ACK using the optimum power level.

We use Shannon capacity as the achievable channel rate,

$$
\text { Channel Capacity }=\mathrm{W} \log 2(1+\mathrm{SIR})
$$

Where $W$ is the channel bandwidth.

\subsection{Algorithm}

Before we introduce the EPCMAC protocol algorithm, it is important to explain the simple diagram shown in Fig. 2. Let $P t[L]$ be a set of the power levels used for the transmission, where $L$ is an integer varies from 1 to $M A X$. Pt [MAX] is the maximum power level and $M A X$ is the number of the power levels in the set. Let $R \operatorname{cs}[L]$ be the set of carrier sensing ranges corresponding to the set of power levels set $P t[L]$.

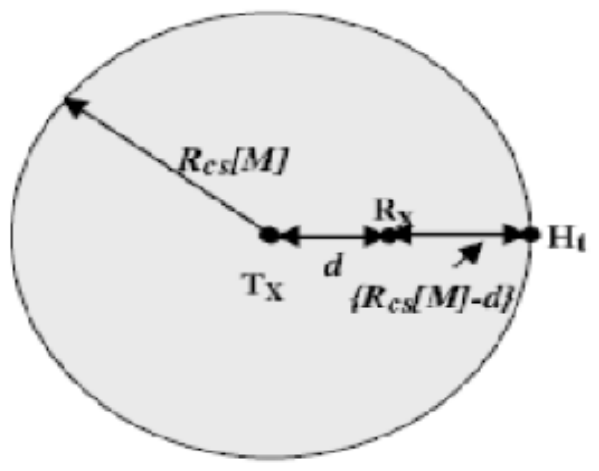

Fig 4: the nearest hidden terminal and the carrier sensing range

From figure 4 let $d$ be the distance between the transmitter $(T x)$ and receiver $(R x)$. Suppose the transmitter reserve a carrier sensing area $R \operatorname{cs}[M]$, where $M$ is an integer variable between 1 and $M A X$. The nearest hidden terminal (Ht) that can be considered as an interference node will be at a distance $\{\operatorname{Rcs}[M]-d\}$ as shown in Fig.2. This carrier sensing range will cover the receiver and $\{R \operatorname{cs}[M]-d\} \geq d$.

\subsection{Algorithm for Transmitter}

i. Let the selected integer value be equal to one that is $\mathrm{L}=1$ and then maximum capacity ratio is given by

\section{Max_Capacity_Area_Ratio=0}

ii. Then the power of the data packets is periodically raised to a suitable level (Pai) to avoid the interference which will be equal to the maximum transmitted power ( $\mathrm{Pt}[\mathrm{MAX}]$ ) which is given by $\mathrm{Pai}=\mathrm{Pt}[\mathrm{MAX}]$

iii. Address of each nodes are checked and its value is stored as $\mathrm{S}$. If the stored value $\mathrm{S}$ is available, then the selected integer value $\mathrm{L}$ will be equal to the stored value $\mathrm{S}$ which is given as $\mathrm{L}=\mathrm{S}$. Send request to send (RTS) packet with the set of the power levels that is used for the transmission (Pt[L]) . 
include the selected integer value $\mathrm{L}$ in the request to send (RTS) packet.

iv. If the request to send (RTS) packet becomes timeout and clear to send (CTS) packet is not received, then the selected integer value $\mathrm{L}$ is varied from 1 to up to 4 . Once the clear to send (CTS) packet is received, its received power $(\mathrm{Pr})$ is observed. Signal to interference ratio (SIR) value is extracted from the clear to send (CTS) packet. Its node address value is stored as $\mathrm{S}$. This stored value $\mathrm{S}$ is assumed to be equal to integer value $\mathrm{L}$ which is given by $\mathrm{S}=\mathrm{L}$

$v$. If the set of the power levels that is used for the transmission $(\mathrm{Pt}[\mathrm{L}])$ is lesser than or equal to the transmitted power level at the crossover distance ( $\mathrm{Pt}, \mathrm{cross})$ then the path loss exponent, $\alpha=2$ in case of free space propagation model and its value is 4 in case of two ray propagation model. $\mathrm{Pt}[\mathrm{L}]<=\mathrm{Pt}$,cross. Determine the distance (d) by using the values of received power $(\operatorname{Pr})$, path loss exponent $(\alpha)$ and set of the power levels that is used for the transmission, $\mathrm{Pt}=\mathrm{Pt}[\mathrm{L}]$ according to Equation (5).

vi. By using the extracted values of Signal to interference ratio (SIR), received power $(\operatorname{Pr})$, path loss exponent $(\alpha)$, set of the power levels that is used for the transmission, $\mathrm{Pt}=$ $\mathrm{Pt}[\mathrm{L}]$, distance (d) and interfering distance, $d i=\{\operatorname{Rcs}[\mathrm{L}]-\mathrm{d}\}$, the interference power $(\mathrm{Pi})$ is determined according to Equation (7).Let $\mathrm{M}=\mathrm{L}$. Where $\mathrm{M}$ is an integer that varies from 1 up to maximum in case of carrier sensing area and $\mathrm{L}$ is an integer value that varies from 1 up to maximum in case of transmission of power levels.

vii. If $\mathrm{M}$ is an integer that varies from 1 up to maximum in case of carrier sensing area is greater than $\mathrm{L}$ which is an integer value that varies from 1 up to maximum in case of transmission of power levels. That is $\mathrm{M}>\mathrm{L}$. Signal to interference ratio (SIR) value is determined according to Equation (7) along with interfering distance $\mathrm{di}=\{\mathrm{Rcs}[\mathrm{M}]-\mathrm{d}\}$

viii. Capacity _Area _Ratio value is determined according to the following Equation

$$
\text { Capacity_Area_Ratio }=\frac{\mathcal{W} \log [(1+\mathcal{S} I \mathcal{R})}{2 \pi *\left(\mathcal{R}_{c S}[\mathcal{M}]\right)^{2}}
$$

ix. If Capacity Area Ratio is greater than maximum Capacity Area Ratio then maximum Capacity Area Ratio is equal to Capacity Area Ratio and the power of the data packets which is periodically raised to a suitable level (Pai) is also equal to the transmitted power $(\mathrm{Pt}[\mathrm{M}])$.

$\mathrm{x}$. If the transmitted power level is lesser than maximum transmitted power level that is $\mathrm{Pt}[\mathrm{M}]<\mathrm{Pt}[\mathrm{MAX}]$ then increase $M$ which is an integer that varies from 1 up to maximum in case of carrier sensing area can be increased up to 11 . This ends the transmitter part.

\subsection{Algorithm for Receiver}

The request to send (RTS) packet is received.

i. Signal to interference ratio (SIR) value is observed and extracted from the received request to send (RTS) packet. Finally its integer value $\mathrm{L}$ is stored.

ii. Signal to interference ratio (SIR) value is then inserted into clear to send (CTS) packet. Clear to send (CTS) packet is transmitted using the set of transmission power level $\mathrm{Pt}[\mathrm{L}]$.This ends the transmitter part.

\section{PERFORMANCE EVALUATION}

The performance of Enhanced Power Control Mac Protocol for Wireless Ad Hoc Networks protocol is analyzed in this section. Simulation is performed using Ns-2 simulator. The parameters used in the simulation is given in table1.Simulation experiments were conducted to assess the performance of our protocol.

Table 1. Simulation parameters

\begin{tabular}{|l|l|}
\hline \multicolumn{1}{|c|}{ PARAMETERS } & VALUES \\
\hline Channel carrier frequency & $2.4 \mathrm{GHz}$ \\
\hline Antenna height & $1 \mathrm{~m}$ \\
\hline Antenna gain & 1 \\
\hline Bandwidth & $2 \mathrm{Mbps}$ \\
\hline CBR traffic rate & $1 \mathrm{Mbps}$ \\
\hline
\end{tabular}

We assumed that the characteristics of all the nodes in the network and the propagation properties are same. The signal propagation model used in our work is a combination of the free space propagation model for near distances and the two ray ground reflection model for far distances. We performed some simulations using different packet size or traffic rate. Table.2 shows the transmit power levels for various transmission ranges considered in the simulation.

The following performance metrics are used to evaluate the MAC protocols.

- Aggregate throughput for the overall flows in the network.

- Data delivered per Joule (Mbits delivered per joule)

- Effective throughput and effective data delivered per joule metrics are used to evaluate the chain topology.

\subsection{Optimal Hop Distance}

When there is a dense collection of immobile nodes we use multi-access multi-hop radio communication with single user decoding and packet forwarding in order to transport packets between various source-destination pairs.

i. All nodes use the same contention mechanism with the same parameters (e.g., all nodes use IEEE 802.11 DCF with the same back-off parameters).

ii. We assume that nodes send control packets (such as RTS/CTS in IEEE 802.11) with a constant power (i.e., power control is not used for the control packets) during contention, and these control packets are decodable by every node in the network. As in IEEE 802.11, this can be done by using a low rate, robust modulation scheme and by restricting the diameter of the network. This is the "single cell" assumption and implies that there can be only one successful ongoing transmission at any time.

iii. During the control packet exchange, each transmitter learns about the channel "gain" to its intended receiver, and decides upon the power level that is used to transmit its data packet. For example, in IEEE 802.11, the channel gain to the intended receiver could be estimated during the RTS/CTS control packet exchange. Such channel information can then be used by the transmitter to do power 
control. In our paper, we assume that such channel estimation and power control is possible on a transmission-by-transmission basis.

iv. In this work, we model only an average power constraint and not a peak power constraint.

v. Saturation assumption: We assume that the traffic is homogeneous in the network and all the nodes have data to send at all times; these could be locally generated packets or transit packets.

We compare the performance of our optimization strategy, i.e., to optimize both the hop distance and power, with simple policies. We report, through extensive simulations, scenarios where it is useful to optimize both the hop distance and power. We compare our optimization strategy, OHOP (Optimal Hop distance and transmit Power for a fading channel), with

i. SHOP (Single Hop direct transmission between source-destination pairs with Optimal transmit Power for a fading channel)

ii. OHCP (Optimal Hop distance with Constant transmit Power in each hop) and

iii. SHCP (Single Hop direct transmission between source-destination pairs with Constant transmit Power).

\subsection{Simulation Results}

EPCMAC achieves a higher aggregate throughput compared to all other schemes. This is because, EPCMAC uses a smaller carrier sensing range compared to $\mathrm{OHOP}$ and $\mathrm{OHCP}$ schemes, since a larger number of nodes can transmit concurrently. Also this carrier sensing range is larger than the carrier sensing range of the OHOP scheme, and it is sufficient to reduce the hidden terminal problem. OHOP and OHCP schemes achieve comparable aggregate throughput as they reserve similar carrier sensing ranges, but the SHOP scheme performs poorly.

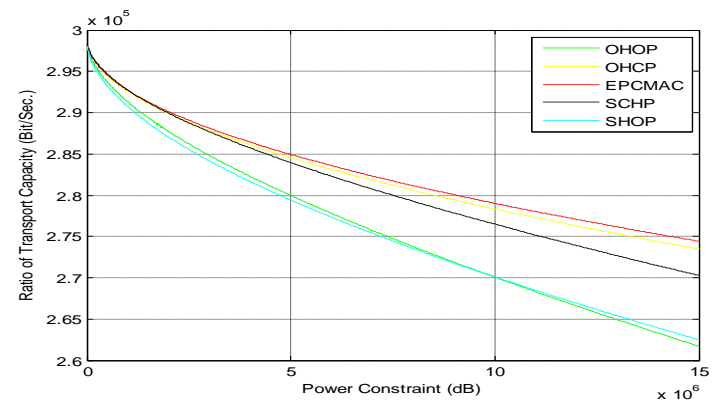

Fig 5: channel average power gain for the capacity of $3 \times 10^{5}$

Figure 5 shows the ratio of the maximum transport capacity with OHOP, OHCP, SCHP, SHOP and the maximum transport capacity achieved using EPCMAC. We consider four different network scenarios, including different network densities, $\eta$ and Rayleigh fade power gain $\mathbf{E}[H]$. We observe that in all the four cases, the ratio of the transport capacity of EPCMAC is at best. Power control is also helpful to counter the effect of fading. The reason is a large number of nodes can transmit simultaneously as the distance increases.

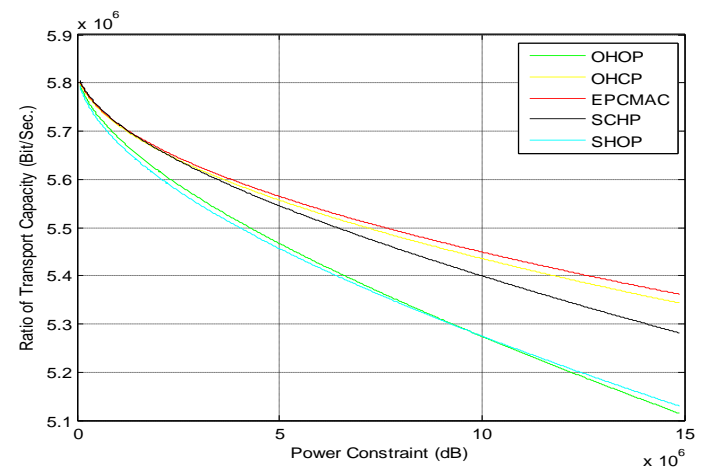

Fig 6: channel average power gain for the capacity of $5.9 \times 10^{6}$

Channel average power gain for the capacity of $5.9 \times 10^{6}$ is shown in figure 6 . The ratio of the maximum transport capacity and the maximum transport capacity of EPCMAC is achieved using the single hop strategy. Clearly, EPCMAC performs much better than OHCP as we optimize over hop distance as well. Also we observe that the performance ratio approaches unity as the time average transmit power increases. As the network power increases, equivalently, the optimal hop length increases and, hence, for large network powers, direct transmission becomes optimal. However, for low values of the available average power, we observe that it is better to optimize over both the hop distance and power.

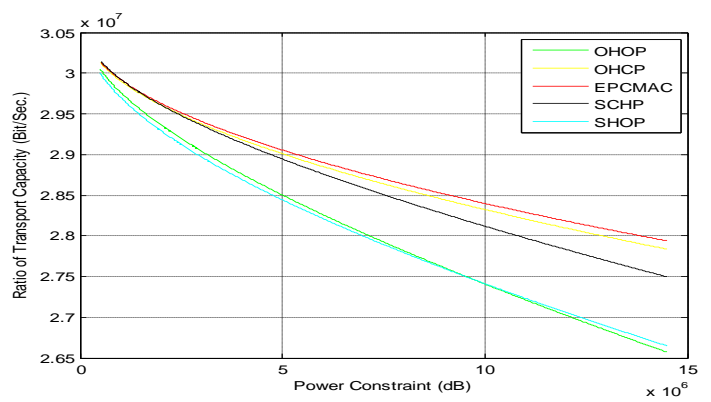

Fig 7: channel average power gain for the capacity of $3.05 \times 10^{7}$

This figure 7 shows the channel average power gain for the capacity of $3.05 \times 10^{7}$ between OHOP, OHCP, SCHP, SHOP and EPCMAC scheme. As the number of data increases, the number of packets successfully delivered is less in all other schemes compared to EPCMAC scheme with power constraint. The performance of EPCMAC with respect to $\mathrm{OHCP}$ improves as the network density increases. Large node densities help the distance discretization technique in tracking optimum distance accurately, thus improving the transport capacity.

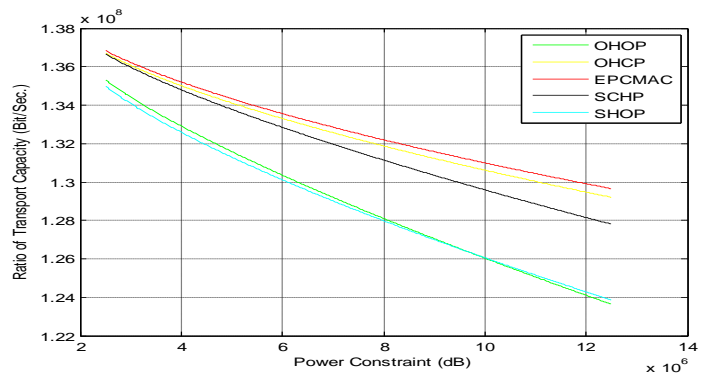

Fig 8: channel average power gain for the capacity of $1.38 \times 10^{8}$ 
The EPCMAC scheme delivers much more data per second compared to all the other schemes as shown in Figure 8. This means that the EPCMAC protocol is effectively better than the others. The EPCMAC scheme achieves a much better aggregate throughput than the others. The aggregate throughputs of the OHCP and EPCMAC schemes are quite comparable, but the SCHP and SHOP scheme performs poorly. The difference in performance between EPCMAC and OHCP is a function only of the optimal bit rate achieved at the optimal hop distance and is independent of the network power. From extensive simulations, we observe that this difference is small in comparison with OHCP for a variety of network scenarios.

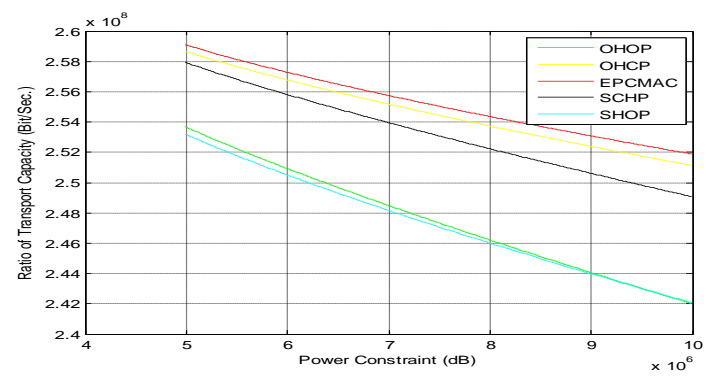

Fig 9: channel average power gain for the capacity of $2.6 \times 10^{8}$

Figure 9 explains the channel average power gain for the capacity of $2.6 \times 10^{8}$ in comparison with $\mathrm{OHOP}$, OHCP, SCHP, SHOP and EPCMAC. EPCMAC scheme provide a characterization of the optimal hop distance and optimal power for cases in which the fading density satisfies a certain monotonicity condition. From extensive simulations, we observe that, for dense ad hoc networks, optimizing the hop distance is essential to maximize transport capacity, especially for low average network powers thereby increasing the performance the EPCMAC scheme compared to all other schemes.

\section{CONCLUSION}

The performance of the EPCMAC scheme with the OHOP, OHCP, SCHP and SHOP schemes is much better compared to other schemes. Its performance under different network scenarios, different packet sizes and various routing protocols is comparatively better. Our simulation results showed that the EPCMAC scheme achieved more total data delivered per second. This means that the EPCMAC scheme can achieves a high reduction in the energy consumption. On the other hand, the simulation results also indicate that the EPCMAC scheme highly improves the network throughput compared to all other schemes. The EPCMAC protocol is mainly designed for single cell, dense wireless ad hoc networks to avoid the interference, save energy and improve the throughput. This reduces the number of unnecessary back-off nodes and allows successful concurrent but interference-limited transmissions to take place in the neighborhood of a receiver. The simulation results also indicate that the EPCMAC scheme highly improves the network throughput compared to all other schemes.

As future work, we intend to perfect the transmission power algorithms in order to avoid frequent transmission power variations. Also, results showed that the use of different preamble sizes and various preamble identification algorithms might decrease packet losses. Finally, the transmission power control technique must be evaluated using chain topology and linear topology which employ channel reservation, exploring RTS and CTS messages to improve the performance of the transmission power calculation.

\section{REFERENCES}

[1] Venkatesh Ramaiyan, Anurag Kumar, Fellow, IEEE and Eitan Altman, Fellow, IEEE, "Optimal hop distance and power control for a single cell, dense, ad hoc wireless network," in IEEE transactions on mobile computing, accepted for publication, march 2011.

[2] Kuei-Ping Shih, Member, IEEE, Yen-Da Chen, and Chau-Chieh Chang, "A physical/virtual carrier-sensebased power control MAC protocol for collision avoidance in wireless ad hoc networks", IEEE transactions on parallel and distributed systems, vol. 22, no. 2, february 2011.

[3] Wei Wang, Student Member, IEEE, Vikram Srinivasan, Member, IEEE, and Kee-Chaing Chua, Member, IEEE, "Power control for distributed mac protocols in wireless ad hoc networks," in IEEE transactions on mobile computing, vol. 7, no. 8, august 2008 .

[4] Kuei-Ping Shih and Yen-Da Chen, "CAPC: a collision avoidance power control mac protocol for wireless ad hoc networks", IEEE communication letters, vol. 9, issue 9, sept. 2005, pp. 859-861.

[5] Xue F, Xie L and Kumar P R, "The transport capacity of wireless networks over fading channels", IEEE transaction on information theory, vol. 51, issue 3 , march, 2005.

[6] Behrouz A. Forouzan, "Data communications and networks", third edition,tata McGraw9Hill edition.2004.

[7] D. Qiao, S. Choi, A. Jain and K. G. Shin, "Adaptive Transmit Power Control in IEEE 802.11a wireless lans", in proceedings of IEEE VTC 2003-spring, jeju, korea, april, 2003, pp.22-25.

[8] F. Ye, S. Yi and B. Sikdar, "Improving spatial reuse of IEEE 802.11 based ad hoc networks", in proceedings of IEEE global telecommunications conference (GLOBECOM), vol. 2, 2003, pp. 10131017.

[9] A.Muqattash and M. Krunz, "Power controlled dual channel (PCDC) medium access protocol for wireless adhoc networks", in IEEE INFOCOM, april 2003, vol.1, pp.4709480.

[10] E.S. Jung and N. H. Vaidya, "A power control mac protocol for ad hoc networks", in ACM MOBICOM, 2002, pp.36-47.

[11] J.Monks, V.Bharghavan and W. Hwu ,"A power controlled multiple access protocol for wireless packet networks", in IEEE INFOCOM, april.2001, pp. 2199228

[12] J.Gomez et al., conserving transmission power in wireless ad hoc networks, proceedings of IEEE 9th int'l. conf. net. protocols, nov. 2001, pp. 24934. 